\title{
XXI.
}

\section{Ueber die physiologische Grundlage der hysterischen 0varie.}

\author{
Von \\ Dr. Steinhausen, \\ oberstabsarzt in Hannover.
}

Zwischen Gesundheit und functioneller Neurose liegt ein breites strittiges und nach unseren heutigen Kenntnissen nicht genau zu definirendes Grenzgebiet. Besonders für die Hysterie hat die Frage der unteren Abgrenzung stets ein gewisses praktisches wie theoretisches Interesse beansprucht, und es fehlt daher nicht an darauf hinzielenden Versuchen. Möbius' Satz ${ }^{21}$ ), „dass jeder Mensch mehr oder minder hysterisch sei", kann nicht mehr als ein geistreiches Paradoxon bedeuten, welches freilich im Hinblick auf die mannigfachen und oft schwer erkennbaren Uebergänge etwas Bestechendes für sich hat. Aber wenn wir auch die Grenze häufig uicht deutlich ziehen können, so hört die Bestimmung derselben doch niemals auf, ein logisches wie nosologisches Postulat zu sein. Dass aber wiederum solche Grenzfragen in enger Wechselbeziehung zu der jeweilig herrschenden Grundanschauung von dem Wesen der Hysterie stehen, liegt auf der Hand, und es wird daher auch die heute verbreitetste Anschauung von der psychischen Herkunft der hysterischen Krankheitserscheinungen auf die Art der Scheidung zwischen Norm und Neurose einen bestimmenden Einfluss haben. Jedenfalls scheint es, dass die Grenze nicht überall so läuft, wie bisher angenommen wird: sie fällt z. B. sicher zum Theil noch in den Bereich der sogen. Stigmata. Der Streit über die Natur derselben ist keineswegs ausgetragen. Bekanntlich sollen die Stigmata - ein Begriff, der übrigens selbst nach Inhalt und Umfang schwankt - mehr oder weniger ausgesprochen in allen Fällen von Hysterie vorhanden sein, gewöhnlich dem Kranken unbewusst bestehen und erst gelegentlich der darauf gerichteten Untersuchung bekannt werden. So werden sie, gegenüber den höher entwickelten und in ihrem Bestande wechselnden Erscheinungen, 1. als Dauersymptome und 2. als eine Gattung niederer Symptome aufgefasst, deren Natur noch von vielen Seiten als rein somatisch angesehen wird ${ }^{22}$ ). Sind sie das, dann bilden sie für das Bestreben, die Aetiologie der Hysterie als einheitlich psychogen anzusehen, eine dauernde Fessel 
und verlangen stets Compromisse bezüglich der Zusammensetzung des Gesammtbildes aus zum Theil der Hysterie nicht wesensverwandten Elementen.

Auf der anderen Seite haben verschiedentlich angestellte Untersuchungen ergeben, dass eine Anzahl der Stigmata auch bei Gesunden so häufig zu finden ist, dass die Fragestellung, ob dieselben somatischer oder psychischer Natur seien, unzureichend ist und nicht den Kern trifft; es muss vielmehr gefragt werden, wie viel an jedem einzelnen Stigma in den Bereich der Norm fällt, wie viel daran pathologisch sei. Man gelangt dann dahin, zu trennen: einmal ein somatisches und physiologisches Grundelement, von dem sich dann nicht mehr behaupten lässt, es gehöre zur latenten Hysterie, und zum Anderen die durch die Hysterie erst bedingte psychogene und pathologische Steigerung.

Die einem hauptsächlichen Theil der Stigmata, den hysterogenen Zonen, zugehörige sogenannte Ovarie ist von mir zum Gegenstand einer Untersuchung gemacht worden, deren Ergebniss nachstehend wiedergegeben sei. Das Ergebniss ist auch unabbängig von mir nachgeprüft worden, und möchte ich an dieser Stelle nicht verfehlen, den Herren Assistenzärzten Dr. Bock und Dr. Schünemann und den einjährigfreiwilligen Aerzten Dr. Wanker und Nordmann meinen Dank für ihre bereitwillige Mitarbeit auszusprechen.

Schon immer war es mir fraglich, ob die durch mehr oder weniger plötzlichen und stärkeren Druck auf die seitlichen Unterbauchgegenden Hysterischer hervorgerufenen Reactionserscheinungen - sofern nicht geradezu ein hysterischer Anfall dadurch ausgelöst wurde - den ihnen allgemein beigelegten diagnostischen Werth beanspruchen können, und ich wurde in diesem vielleicht auch anderen Beobachtern aufgestossenen Zweifel sogleich bestärkt, als ich die vergleichende Probe bei Gesunden machte. Und als nicht besser geeignet solche Bedenken über den Werth der Ovarie zu beseitigen, erwies sich ein Rückblick auf die Geschichte derselben. Eine kurze Uebersicht der Wandlungen, die ihre Auffassung im Laufe der Zeit erfahren hat, möge hier zunächst folgen.

Vorher bereits gekannt, wurde die Ovarialgie zuerst von Piorry (1837) und Schützemberger (1846) in unmittelbare und von letzterem Autor sogar in ursächliche Beziehung zur Hysterie gesetzt (Chez les femmes, l'excitation ovarique est la cause la plus fréquente des perturbations hystériques 1). Piorry unterschied bei hysterischen Frauen drei bei D ruck auf die Ovarialgegend hervorgerufene Phasen: 1. schmerzhafte Irradiation zum Epigastrium, 2. Herzpalpitationen mit Steigerung der Pulsfrequenz und bei längerem Druck schliesslich 3. Erscheinungen des Globus. Von älteren Autoren sei ferner Briquet ${ }^{2}$ ) angeführt, 
welcher die Bauchmuskeln als Sitz der "Coelialgie" annahm und damit eine eigentliche Ovarie schon damals in Abrede stellte.

Der Begründer aber der heutigen Lehre von der Ovarie ist auf Grund seiner ausgedehnten Beobachtungen an Hysterischen Charcot geworden. Er glaubte an der typischen Stelle durch Druck den Eierstock zu reizen, sah als Ursache der Ovarie das hyperästhetische Ovarium an und grenzte als ovarielle Hysterie geradezu eine besondere Form ab. Diesen Anschauungen findet sich auf fast jedem Blatt der klinischen Vorlesungen Ausdruck gegeben, und spätere Einschränkungen dürften auf das Fehlen jeder anatomischen Veränderung an den Ovarien bei der Section, wie dies auch von Richet, Westphal u. A. bestätigt wurde, und namentlich auf die Misserfolge der Ovariotomie zurückzuführen sein. Als weitere typische Phasen, ausser denen Piorry's, bezeichnet Charcot eine Reihe von Sensationen (Zischen im Ohr, Hammerschläge gegen die Schläfe etc.), sodann Umnachtung und schliesslich Bewusstlosigkeit und Krämpfe. Charcot klagt gelegentlich darüber, dass die Ovarie ausser von Romberg seitens der deutschen Autoren ignorirt worden sei, und in der That sind gerade von dieser Seite die ersten schwerwiegenden Einwände gegen seine Lehre von der Ovarie erhoben worden. Konnte schon der engen Zusammengehörigkeit der Tvarie mit der Hemianästhesie im Sinne Charcot's in Deutschland nicht zugestimmt werden, so wurden namentlich auch Einwände gegen den Sitz und Ursprung der Ovarie erhoben. So hatte Valentiner Hyperästhesie des Bauchfells, Schröder van der Kolk einen Krampf des Colons als Ursache angesprochen, während Arndt als einer der Ersten betonte, dass ebendieselbe Ovarie auch bei männlicher Hysterie vorkomme und daher unmöglich mit den Eierstöcken in Beziehung zu bringen sei. Anfang der achtziger Jahre wiesen Schultze ${ }^{3}$ ), Hasse ${ }^{4}$ ) und später Schroeder ${ }^{5}$ ) und Gusserow $\left.{ }^{6}\right)$ nach, dass bei der von Charcot angegebenen Methode die Eierstöcke äberhaupt nicht getroffen werden können. Inzwischen war in zahlreichen Fällen zur Heilung schwerer Hysterie die operative Entfernung der Ovarien vorgenommen worden, ohne dass ein wirklicher Erfolg erzielt wurde. Zur Entscheidung der brennend gewordenen Frage theilten u. A. Landau und Remak ${ }^{7}$ ) einen ebenso typischen wie beweiskräftigen Fall mit, in welchem auch nach der Ovariotomie die Erscheinungen der Hysterie unverändert fortbestanden und es sich nicht um ovarielle Hysterie, sondern hysterische Ovarie handelte, daher die Operation zu keiner Zeit angezeigt gewesen war. Die Verfasser betonten in Anschluss an diese prägnante Erfahrung, dass die Ovarie nicht nur nicht die Ursache der Hysterie sein könne, dass vielmehr Ovarial-Hyperästhesie auch als selbständiges Leiden 
vorkomme und als Theilerscheinung der Hysterie überhaupt gar nicht so häufig sei. Auch Jolly ${ }^{8}$ ) bestritt die Häufigkeit dieses Vorkommens und stellte die Ovarie auf eine Stufe mit den sonstigen Hyperästhesien.

An der Bedeutung der Ovarie im Charcot'schen Sinne scheint seitens der französischen Autoren allgemein bisher festgehalten zu werden. Um nur einen der bekanntesten anzufăhren, sieht Gilles de la Tourette ${ }^{9}$ ) darin eins der constantesten Cardinalsymptome und die wirksamste hysterogene Zone, deren Sitz zweifellos das Ovarium sei. Da aber die Charcot'sche Ovarie der männlichen Hysterie nothwendigerweise fehlt, so erkennt Gilles de la Tourette beim Manne eine Pseudo-Ovarialzone an, deren Sitz keinesfalls die Hant, für die es aber fraglich sei, ob sie den visceralen Zonen zuzurechnen sei, da ihr beim Manne kein besonderes Organ entspreche.

Zur Zeit liegt bei uns die Sache so, dass seitens der Gynäkologen am meisten durchgreifend die hysterische Ovarie von der als selbständiges Leiden auftretenden echten Ovarialhyperästhesie getrennt wird (Olshausen, Kleinwächter, Apostoli u. A.). In der neurologischen literatur findet sich fast durchgehend die Frage sowohl nach den Bexiehungen der hysterischen Ovarie zu den Eierstöcken, als auch nach der Ursache des analogen Vorkommens beim Manne und damit nach dem eigentlichen Sitz derselben als eine noch offene behandelt. Mit der Annahme der Testikelhyperästhesie als des Analogons der Ovarialgie geht man vielfach der Beantwortung aus dem Wege (z. B. Hirt $\left.{ }^{10}\right)$ ). Einzelne Arbeiten, die sich u. A. auch mit dem Sitz und Ursprung der Ovarie beschäftigen, kommen zu widersprechenden und jedenfalls nicht überzeugenden Ergebnissen; so finden sich nach Lomer ${ }^{11}$ ) bei Genitalerkrankungen der Frauen hyperästhetische Hautzonen am häufigsten in der Ovarialgegend, doch lasse sich die Ovarie nicht auf bestimmte Hautnerven beziehen. Um noch einige andere Urtheile anzuftuhren, glaubt auch Breuer ${ }^{12}$ ) (S. 165) sie von Zuständen des Genitalapparates abhängig; Gowers ${ }^{13}$ ) erklärt den Ovarialschmerz für tiefsitzend. Nach K y ri ${ }^{14}$ ) sind es bestimmte Nervenbahnen, auf welchen Erkrankungen der Ovarien nach aussen auf die Bauchhaut projicirt werden und welche auf Reizung durch Druck hin Ovarie hervorrufen. Die im Uebrigen ja vortrefflichen Untersuchungen Head's geben über die in Rede stehende Frage keine verwendbaren Aufschlüsse, da es sich bei den in Betracht kommenden 8. bis 11. seiner topograpischen Zonen um Veränderungen der Hautsensibilität bei Erkrankungen der Baucheingeweide, namentlich der Geschlechtsorgane handelt.

Jedenfalls figurirt die Ovarie nach wie vor allgemein als eines der wichtigsten und am meisten charakteristischen Dauersymptome der 
Hysterie. Giebt es z. B. nach Ziehen ${ }^{15}$ ) keine Hysterie ohne alle hysterogenen Punkte, so steht unter diesen die Ovarialzone obenan. In neuester Zeit geht wohl am weitesten in der diagnostischen Verwerthung der Ovarie Windscheid ${ }^{16}$ ); sie bildet nach ihm zusammen mit dem Fehlen des Bindehautreflexes und der Steigerung des Patellarreflexes die für die Diagnose der Hysterie grundlegende Symptomentrias. Gleichwohl hat aber merkwürdigerweise gerade Wind scheid mit Teich müller festgestellt, dass die Ovarie auch bei anderen Neurosen und bei nervöser Exaltation, ja auch sogar bei nicht nervösen Krankheiten vorkommt, und dass hier sich Männer in bedeutender Ueberzahl finden. Es verdient noch erwähnt zu werden, dass auch Gowers ${ }^{13}$ ) Empfindlichkeit der Unterbauchgegend auch bei nicht hysterischen Frauen keineswegs selten gefunden hat.

An Gesunden sind meines Wissens ausgedehntere Untersuchungen betreffs des Vorkommens von Erscheinungen der Ovarie bisher nicht angestellt worden.

Was die praktische Verwendung der Ovarie zu diagnostischen Zwecken betrifft, so wird sie heute, soweit mir bekannt, ziemlich allgemein und mehr oder weniger regelmässig in die Gesammtuntersuchung hineinbezogen, und es werden als abortive oder anfallähnliche $\left(Z_{i e h e n}{ }^{15}\right)$, Freund $\left.{ }^{12}\right)$ ) auch die leichten durch Druck auf die bekannte Stelle hervorgerufenen Erscheinungen zur Bestätigung der Diagnose der Hysterie verwerthet. Eine nähere Definition des für die Diagnose etwa erforderlichen oder die untere Abgrenzung des Paroxysmus etwa bestimmenden Grades der Erscheinungen existirt bisher jedenfalls nicht.

Es erhellt, dass zur Feststellung von Ovarieerscheinungen bei Gesunden nur von der Prüfung einer grossen Anzahl möglichst vollkommen normaler Individuen Aufschluss zu erwarten ist. Besitzen wir auch keinen absolut sicheren objectiven Anhalt, wonach wir entscheiden könnten, ob ein Nervensystem als gesund zu bezeichnen sei, so lassen sich doch eine Anzahl Bedingungen erfüllen, um ein einigermassen verwerthbares Resultat zu gewinnen. Sodann ist aus bekannten Gründen die Untersuchung beim männlichen Geschlecht vorzuziehen. Ein derartiges sicher auszuwählendes Untersuchungsmaterial bot sich mir in den Mannschaften des activen Dienststandes der Armee. Schon die bei der Einstellung geübte Auslese, das jugendliche Alter, die gesunde körperliche Thätigkeit, das von Sorgen in der Regel nicht wesentlich getrübte Dasein, Alles das bietet die ziemlich weit gehende Garantie. Weitere Gewähr liegt in der Ausschliessung aller derjenigen Leute, bei denen ererbte neuropathische Anlage im Laufe der Dienstzeit in irgend einer Weise in die Erscheinung ge- 
treten ist, ferner auch solcher, welche durch den plötzlichen Uebergang aus den heimischen Verhältnissen in die straffe militärische Erziehung, durch die Loslösung aus der gewohnten Umgebung und das Einwirken neuer starker Eindrücke an Frische und Elasticität eingebüsst haben [Düms $\left.\left.{ }^{20}\right)\right]$. Sodann war geboten, namentlich auch die Aussonderung aller körperlich nicht absolut Gesunden und schliesslich die Wahl eines Zeitpunktes für die Prüfung, welchem übermässige und erschöpfende Anstrengungen nicht vorausgegangen waren. Günstig war auch der Umstand, dass die Mannschaften, an häufige Untersuchungen gewöhnt, in der fraglichen Prüfung keinen ausserordentlichen und irgendwie erregenden Vorgang erblickten. Bei den in kleineren Gruppen und isolirt vorgenommenen Untersuchungen wurde selbstredend jede suggestive Beeinflussung ausgeschlossen, nur das Nöthigste bezüglich der subjectiven Empfindungen gefragt und jedes Hineinexaminiren vermieden. Das manuelle Ausüben des Druckes fand auf beiden Seiten der Unterbauchgegend in der bekannten von Charcot u. A. vorgeschriebenen Weise und zwar plötzlich und mit mässigem Kraftaufwand statt. Im Ganzen wurden 500 Leute untersucht und es fanden sich, in Procenten ausgedrückt, für die einzelnen dabei zu beobachtenden Erscheinungen folgende Häufigkeitszahlen:

\section{a. Sensible Erscheinungen.}

1. Unangenehmes kitzelartiges Gefühl in . . . . . . . 66 Proc.

2. Kitzelgefühl mit mehr oder weniger starkem Lachreiz . . 10 "

3. Anfsteigende Empfindung von Hitze oder Beklemmung . . 7 ,

4. Schmerzempfindung . . . . . . . . . . . . . . 13 "

\section{b. Motorische Erscheinungen.}

1. Spannung der Bauchdecken . . . . . . . . . . . . 58

2. Adduction und Streckung der Arme . . . . . . . . 11

3. Seitlich ausweichende Längsdrehung des Rnmpfes . . . . 48

4. Streckung und Ueberstreckung (Opisthotonus) der Wirbelsäule 17

5. Beugung der Wirbelsäule . . . . . . . . . . . . 8

6. Adduction und Einwärtsrollung und Beugung der Beine. . 56

7. Streckung der Beine . . . . . . . . . . . . . . 5 "

c. Psychische Erschelinungen.

1. Schreckhafter Gesichtsausdruck . . . . . . . . . . 38

2. Erotisch-erregter Gesichtsausdruck . . . . . . . . $10 "$

d. Erscheinungen im Gefässsystem.

1. Röthung des Gesichts . . . . . . . . . . . . . 15

2. Vorübergehende Erhöhung der Pulszahl um $8-20$ i. d. Min. 23 " 
e. Erscheinungen seitens der Athmung.

1. Beschleunigung der Athmung . . . . . . . . . . . 6 Proc.

2. Einzelne heftigere Aus- oder Einathmung . . . . . . . 31 "

3. Nicht unterdrückbarer Stridor dabei in den oberen Luftwegen 19 "

$$
\text { f. Pupillenerweiterung. } 81
$$

Davon a. geringen Grades . . . . . . . . . . . . 57 "

b. stärkeren Grades . . . . . . . . . . . . . 24 "

g. Anderweitige Erscheinungen.

Schallender Ructus . . . . . . . . . . . . . . . 12 "

Im Ganzen wurden bei 88 Proc. überhaupt Reactionserscheinungen festgestellt, der Rest verhielt sich reactionslos, auch bei mehrfacher Untersuchung*). Von den 19 der oben aufgezählten Einzelerscheinungen fanden sich bei einem und demselben Individuum höchstens 13 vor.

Es wurden beobachtet:

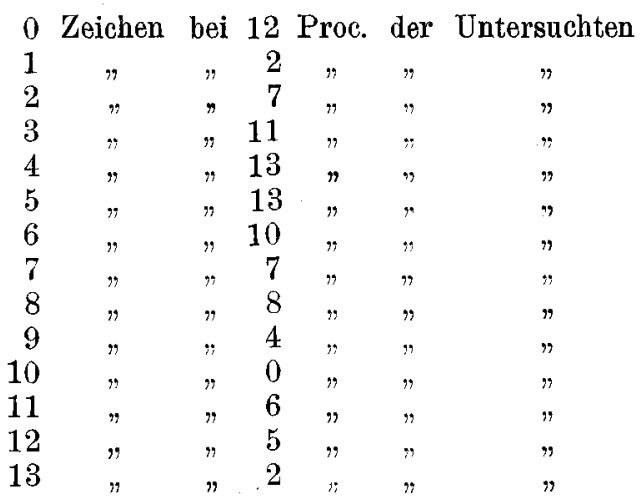

Der Durchschnitt betrug zwischen 5 und 6 und zwar in wechselndster Combination. Auch hinsichtlich des Stärkegrades waren beträchtliche Unterschiede zu verzeichnen, die des Näheren wiederzugeben unmöglich ist, und für deren Schätzung die Nachprüfung den Fachgenossen empfohlen sei.

Zwischen der rechten und linken Seite konnte ein wesentlicher

*) Ich muss hier den bei meinen Untersuchungen gewonnenen Eindruck wiedergeben, dass die militärische Vorgesetztenstellung des Untersuchers bei meinem Material einen nicht unerheblichen Einfluss hat, ein psychischer Factor, der hemmend wirkt und nicht genügend ausuuschalten ist. 
Unterschied nicht festgestellt werden. Bezüglich der sensiblen Erscheinungen sind noch einige Erläuterungen beizufügen. Die Gefühlsbetonung ist ganz überwiegend eine unangenehme, auch da, wo das Kitzelgefühl mit Lachreiz sich rerband. Nähere Nachfragen über begleitende erotische Empfindungen wurden unterlassen. Es ist mir indess nicht unwahrscheinlich, dass diese häufiger bestehen, als sie in den obigen Zahlen erscheinen. Hier mag erwähnt sein, dass bei gesunden Frauen, die ich allerdings nur in einer kleinen Anzahl zu untersuchen Gelegenheit hatte, Lachreiz trotz der stets unangenehmen Begleitgefühle noch weit häufiger zu beobachten war; auch wurde es von ihnen als unangenehm empfunden, dass sie diesen Reiz nicht zu unterdrücken vermochten.

Die motorischen Erscheinungen dürften im Wesentlichen als Abwehr- oder Ausweich-Bewegungen anzusehen sein, nur bei den Streckbewegungen der Wirbelsäule (Opisthotonus) scheint mir diese Erklärung im Stich zu lassen und vielleicht auch bei manchen der sehr verschiedenartigen Bewegungen der unteren Gliedermassen. Sie gewähren auch in mehr oder weniger ausgesprochener Weise den Eindruck reflectorischer Muskelcontractionen, deren Zweckmässigkeit nicht immer einzusehen ist. Ihrer Dauer nach sind dieselben als tonisehe zu bezeichnen.

Eine Reihe vergleichender Prüfungen ergab, dass kein wesentlicher Unterschied besteht, ob der Druck auf den Unterleib vorher angesagt war oder nicht, wie denn auch das einzelne Male zum Vergleich voraufgegangene Zuschauen bei einer Untersuchung keinen erkennbaren Einfluss zeigte. Es dürfte sich hierbei um Factoren psychischer Art handeln, die möglicherweise bei anderem Untersuchungsmaterial in mehr oder weniger abweichender Gestalt sich geltend machen werden. Sicher ist aber, dass ein schwach einsetzender, nur sehr allmählich gesteigerter und langsam ablaufender Druck ganz ungleich geringere Wirkung*) hervorruft, als der plötzliche, eine Beobachtung, die bezeichnenderweise auch bei der Auslösung ovarieller hysterischer Anfälle von jeher gemacht worden ist.

Dem plötzlichen Druck tritt wie abwehrend und dem tieferen Eindringen direct hinderlich die tonische Spannung der Bauchdecken entgegen, welche durch den Willen insofern vermindert werden kann, als bei längerem und nicht schmerzhaftem Druck auf Einreden hin Erschlaffung zu erzielen ist. Der Eindruck, den diese Art von "Schutzvorrichtung" macht, welche ja bekanntlich dem tieferen Eindruck an jeder Stelle des Unterleibes bei der Palpation z. B. der Leber

*) vgl. Str ü mpell $\left.{ }^{19}\right)$. 
und Milz hindernd entgegentritt, lässt nicht schlechthin den Schluss $\mathrm{zu}$, dass der Sitz der sogen. Ovarie etwa die Bauchhaut oder die Bauchmuskeln seien. Von dem Bauchreflex unterscheidet sich der Vorgang schon durch den tonischen Charakter und durch den Einfluss des Willens. Bekanntlich gehen, wie schon erwähnt wurde, die Meinungen über diesen "Sitz" auseinander. Für einen solchen in der Tiefe und nicht in der Haut sprechen folgende Umstände: 1. Blosse Reizung der Haut, z. B. Kneifen einer Hautfalte, Reizung durch Stich u.s.w. pflegt nur die bekannte Reflexzuckung in der Bauchmusculatur zur Folge zu haben. 2. Auch leichter blos die Bauchdecken treffender Druck bringt Spannung derselben hervor, genügt aber niemals zum Auslösen aller Erscheinungen. 3. Die Angaben der darüber befragten Untersuchten verlegen übereinstimmend den Ausgangspunkt aller sensiblen Erscheinungen in die Tiefe der Bauchhöhle, ohne indess die fragliche Stelle irgendwie genauer localisiren zu können. Der Vollständigkeit halber mag nicht unerwähnt bleiben, 1. dass niemals die geringste Schädigung durch den plötzlichen Druck bedingt wurde, 2. dass niemals spontaner Schmerz an der betreffenden Stelle bestand, und 3. dass Herabsetzung der Sensibilität der Haut bei den Untersuchten niemals gefunden wurde, ebensowenig wie Hyperästhesien (Head) und Hyperalgesien*).

Es wurde ferner das Verhalten der Reactionserscheinungen bei mehrmaliger Wiederholung der Untersuchung geprüft. Was zunächst die Spannung der Bauchdecken betrifft, so trat dieselbe in der Mehrzahl bei jeder Wiederholung gewöhnlich eher etwas gesteigert auf, dagegen liessen die übrigen motorischen Erscheinungen bald geringe Zunahme, bald Verringerung erkennen, so dass eine Regel in dieser Beziehung nicht abzuleiten war. Hier spielen die verschiedensten psychischen, der Controle sich entziehenden Einflüsse hinein. Nicht selten hatte auch der scheinbare, nur beabsichtigte, aber dann nicht ausgeübte Druck schon ähnliche Abwehrbewegungen zur Folge. Zweifellos wirkt der Wille, wie ja von vornehein anzunehmen, hemmend ein, jedoch scheint es grösserer Energie zur Abschwächung oder völliger Unterdrückung zu bedürfen, als man denken sollte.

Sprechen bezüglich der Localisation der reizaufnehmenden Stelle die beschriebenen Erhebungen für eine solche im Innern der Unter-

*) Halbseitige Herabsetzung und Erhöhung der Hautsensibilität ist bis zu einem gewissen Grade auch bei suggestiblen gesunden Individuen hervorzurufen. Ich möchte deshalb denjenigen Autoren, wie z. B. Bötticher ${ }^{17}$ ), beistimmen, welche der Ansicht sind, dass diese Erscheinungen auch bei nicht darauf untersuchten Hysterischen nicht vorkommen, bezw. in einer für den Kranken selbst der Nichtexistenz gleichkommenden Weise latent bleiben. 
leibshöhle, so sind wir bezüglich der anatomischen Verhältnisse nur auf Vermuthungen und Schlussfolgerungen aus anderweitigen Erfahrungen angewiesen. Indess dürfte dasjenige, was wir über die Endigungen der sympathischen Nerven im Bauchfell und in der Darmwandung und die Einflechtung sensibler und motorischer spinaler Elemente in die sympatischen Plexus wissen, der Annahme nicht entgegensteben, dass es sich für die Vermittlung der Ovarieerscheinungen nur um die visceralen Verzweigungen des sympathischen Nervensystems handeln kann. Sind es im Wesentlichen 3 Gruppen, in welche die oben beschriebenen Erscheinungen zusammengefasst werden können: motorische, sensible und sympathische, so spricht gerade die Grösse des Antheiles, welcher den speciell auf Sympathicus-Reizung zu beziehenden Erscheinungen, seitens der Pupille, der Athmung, des Gefässsystems u. s. w. zukommt, für die Richtigkeit jener Annahme. Analoge Vorgänge liegen zu Grunde: 1. der bekannten schon von Erb, Moeli und Westphal beobachteten Thatsache, dass bei jeder stärkeren sensiblen Hautreizung bei Gesunden Pupillenerweiterung eintritt, und 2. dem Mannkopff-Rumpf'schen Zeichen, der Steigerung der Pulsfrequenz bei allen stärkeren sensiblen Reizen (vgl. Oppen heim ${ }^{18}$ ) S. 813).

Das Gesammtergebniss aus den Untersuchungen betreffend das Vorkommen von "Ovarie" bei Gesunden, erscheint in mehrfacher Hinsicht bemerkenswerth. Berücksichtigt man die Constanz und den Grad der Erscheinungen und ihre Häufigkeit bei 88 Proc. vollkommen normaler Menschen, so ergiebt sich daraus der Schluss, dass es sich uur um rein physiologische Vorgänge handeln, und dass diese Art "Ovarie" an sich keine Beziehungen zur Hysterie hat.

Im Anschluss hieran drängen sich nun folgende Fragen auf.

I. Welcher Gattung physiologischer Erscheinungen würden die bisher als Ovarie bezeichneten Vorgänge anzureihen sein, und welches ist somit das Wesen derselben?

Zunächst kann darauf hingewiesen werden, dass die hysterische Ovarie allerseits und von jeher als eine Summe krankhafter reflexartiger Phänomene aufgefasst worden ist. $\mathrm{Da}$ ferner bisher eine Trennung der hysterisehen Ovarie von den ihr zu Grunde liegenden physiologischen Erscheinungen nicht gemacht worden ist, so gilt das Gleiche auch von der "Ovarie" bei Gesunden, und in analoger Weise wird also auch diese den complicirten zum Theil durch den Willen mehr oder minder beeinflussbaren Reflexphänomenen zugerechnet werden müssen. Es ist bemerkenswerth, dass als zu dieser Gruppe gehörig auch die Ausweich- und Abwehrbewegungen auf Kitzelreize 
angesehen werden $\left.\left(\mathrm{Gad}^{23}\right)\right)$. Nach dem bei meinen Untersuchungen gewonnenen Gesammteindruck hat sich mir denn auch in der That von Anfang an die Analogie der sog. Ovarie in ihrer eigenthümlichen Combination sensibler und motorischer Phänomene mit dem Hautkitzel aufgedrängt. Eine Anregung gab mir in dieser Hinsicht auch eine Unterredung über Erscheinungen des Hautkitzels mit Herrn Collegen Bruns, dem ich auch an dieser Stelle für sein Interesse meinen Dank aussprechen möchte.

Jene Analogie gründet sich im Einzelnen auf folgende Erwägungen. Zunächst spielt das Kitzelgefühl eine ganz überwiegende Rolle unter den oben analysirten sensiblen Erscheinungen, so dass es für die subjective Seite derselben geradezu bestimmend erscheint. Dagegen tritt das Schmerzgefühl mehr in den Hintergrund, es kann höchstens zur Erklärung des begleitenden Unlustgefühls herangezogen werden, wie denn Kitzel und Schmerz ja auch sonst als Gemeingefüble zusammengehören.

Der Kitzel wird bekanntlich als ein Summationsphänomen definirt und ist an sich keine von den Berührungs- und Druckempfindungen verschiedene Qualität (Goldscheider ${ }^{25}$ ), Gad $\left.{ }^{23}\right)$ ). Liegen nun ganz ähnliche Summationsvorgänge dem abdominalen Kitzel, der physiologischen „Ovarie“ zu Grunde, so tritt die Analogie noch sinnfälliger hervor durch den Umstand, dass der Hantkitzel gleichfalls eine besondere Localisation an gewissen Gegenden der Körperoberfläche (Hals, Achselhöhle, Leistenbeuge, Kniekehle etc.) aufweist, Stellen, an denen die grossen Gefässe und Nervenstämme verlaufen. Hier ruft jede Berührung - allerdings in individuell sehr schwankendem Grade - und bisweilen auch blos der Versuch der Berührung eine Reihe von Reactionserscheinungen hervor, welche mit den beschriebenen der "Ovarie" vollkommen übereinstimmen. Es handelt sich also im Wesentlichen um Schutz- und Abwehrbewegungen, welche durch den mehr oder minder bewusst eingreifenden Willen vor den einfachen spinalen Reflexen sich auszeichnen und den Stempel des Zweckmässigen tragen (Strümpell $\left.{ }^{19}\right)$ ).

Trotz dieser Aehnlichkeit besteht dennoch zwischen dem localisirten Kitzel und der sogen. Ovarie ein gewisser Unterschied, welcher sich auf den Sitz, den Ausgangspunkt bezieht. Blosses Kitzeln der Bauchhaut hat nicht entfernt die Wirkung bezüglich der Auslösung der typischen Reactionserscheinungen, wie der tiefe Druck. Insbesondere fehlen auf blossen Kitzelreiz die vasomotorischen Erscheinungen, sowie diejenigen seitens der Athmung, der Pupille etc. Ob den Muskeln und Fascien der Bauchdecken eine Bedeutung für die Entstehung des Phänomens zukommt, erscheint mir zweifelhaft. 
Es wird sonach, in Uebereinstimmung mit den subjectiven Angaben der Untersuchten, der Schluss gerechtfertigt sein, dass die sogen. Ovarie eine in eigenthümlicher Weise localisirte, an den visceralen Inhalt der Unterleibshöhle gebundene Form des Kitzels darstellt, für welche die Bezeichnung "visceraler Kitzel" mir am angemessensten erscheint.

II. Welche Beziehungen bestehen zwischen den physiologischen Ovarieerscheinungen und der Hysterie?

Die bisherige Auffassung der Ovarie als hysterischen Symptoms schlechthin kann nicht mehr als berechtigt angesehen werden. Man müsste denn behaupten wollen, alle die mit den oben beschriebenen Erscheinungen behafteten Leute seien als hysterisch oder wenigstens als latent hysterisch zu bezeichnen. Eine derartige Annahme aber ist bei der geradezu vorbildlichen Gesundheit des Untersuchungsmaterials durch nichts gerechtfertigt, und weder der Wissenschaft noch unserer Nation wäre damit eine Dienst erwiesen.

Das aber soll keineswegs gelengnet werden, dass die sogen. Ovarie, der Visceralkitzel, in bestimmte Beziehungen zur Hysterie treten kann und seinem Wesen nach besonders geeignet ist, an und für sich ein leeres Schema, unter gewissen Bedingungen zu einem hysterischen Symptomcomplex ausgestaltet, gesteigert zu werden. Diese Bedingungen aber schafft die Hysterie erst und im Besonderen die hysterische Individualität. Es ist eine bekannte Thatsache, dass die Hysterie alle möglichen körperlichen krankhaften Zustände gleichsam benutzt und zu einer ihrer Theilerscheinungen modificirt und steigert und sich damit im Einzelfall determinirt (Breuer, Freund u. A.). Daher ja auch die Möglichkeit der zahllosen Combinationen der Hysterie mit wohl allen bekannten organischen Nerven-, Rückenmarks- und Hirnkrankheiten. Besteht also das Wesen der hysterischen Ovarie erst in der krankhaften Steigerung des Visceralkitzels, so ist es erklärlich, dass diese Steigerung die individuell weitesten Schwankungen erfahren und alle Stufen bis zum grossen hysterischen Anfall durchlaufen kann. So findet die interessante Thatsache ihre Erklärung, dass alle Elemente des ausgeprägten hysterischen, durch Druck auf das Abdomen ansgelösten Anfalles in den Erscheinungen des Visceralkitzels bereits im Keime enthalten sind (einschliesslich der Aura, des Globus, der subjectiven Pulsationsgefühle etc.), so dass man geradezu sagen kann, derselbe stelle das physiologische Urbild des hysterischen Anfalles dar. Aller Wahrscheinlichkeit nach kommt, wie ich aus bisher noch nicht abgeschlossenen Untersuchungen schon jetzt glaube ableiten zu können, ähnlichen kitzelartigen $\mathrm{Pbänomen} \mathrm{auch} \mathrm{bei} \mathrm{anderen} \mathrm{hysterogenen}$ Zonen ein gleich wesentlicher Antheil wie bei der "Ovarialzone" zu. 
Diese Zone dürte aber gerade der eigenartigen Constanz der von ihr ansgehenden Reactionserscheinungen ihre hervorragende Stellung in der hysterischen Symptomatologie verdankt haben.

In dieser Auffassung ist die hysterische Ovarie, d. h. also der zum hysterischen Symptom gesteigerte viscerale Kitzel, eine rein psychogene Erscheinung, und es dürfte damit die Frage erledigt sein, wie viel dieselbe an somatischen Elementen etwa aufzuweisen habe. ${ }^{22}$ ) Zur Charakterisirung des Gegensatzes mag über diese Frage die Ansicht von Breuer erwähnt sein (a. a. O. S. 165), welcher die „gewöhnliche Ovarie" für somatischen Ursprungs, die Ausscheidung derselben aus dem hysterischen Symptomencomplex gleichwohl für nicht angängig hält.

III. Aus welchem Grunde sind die physiologischen Erscheinungen der sogen. Ovarie bisher für pathologisch und hysterisch gehalten worden?

Diese Frage ist nur unter der Annahme eines fortlanfenden Irrthums über Wesen und Bedeutung der Ovarie befriedigend zu beantworten.

Den Vorgang vor anderen hysterogenen Zonen hat die Ovarialzone, wie schon erwähnt, zunächst der Constanz und Häufigkeit der an die Iliacalgegend gebundenen physiologischen Reactionserscheinungen, sodann aber ganz besonders dem altgeheiligten Dogma von dem nahen Zusammenhang der Hysterie mit krankhaften Zuständen der weiblichen Generationsorgane zu verdanken. Nur von diesem Gesichtspunkt aus erscheint es verständlich, dass so viel Willkürliches und Subjectives in der Auffassung von dem Wesen der Ovarie sich so lange hat behaupten können. Gewiss ist es nach anderen Erfahrungen auf dem Gebiet der grossen Neurose nichts weniger als zufällig, dass gerade in Frankreich die Lehre von der Ovarie entstanden und so weit herausgearbeitet worden ist. Auch Charcot selbst sind andere ähnliche Irrthümer begegnet, und es geschieht gewiss den von der Geschichte anerkannten grossen Verdiensten des berühmten Forschers kein Abbruch, wenn man eine auf Subjectivität und einer Art von Autosuggestion beruhende Täuschung wie auf dem Gebiet der Hypnose so auch hier einräumt. Auch an das Schicksal, welches die Lehre vom Transfert erlebt hat, kann hier erinnert werden.

In den Bereich rein suggestiver Wirkung muss auch die von Charcot u. A. systematisirte Unterdräckung hysterischer Anfälle durch Ovarialdruck verwiesen werdert. Dass unter den sogen. hysterofrenatorischen Zonen ebenfalls die Unterleibsgegend eine bevorzugte Rolle spielt, kann nach Allem nicht Wunder nehmen.

Drängt sich hier nicht dem unbefangenen Beurtheiler die Ver- 
muthung auf, dass das Dasein der hysterischen Ovarie und mit ihr des entsprechenden durch suggestive Steigerung hervorgebrachten Typus des hysterischen Anfalles in Frage gestellt würde, sobald man das Aufsuchen aller Ovariesymptome zu unterlassen sich entschliessen könnte, ja, dass dieselben vielleicht ganz aus der Welt verschwinden würden? Wie umgekehrt bei einer grossen Anzahl Hysterischer durch öfter wiederholte Untersuchungen die Ovarie zum dauernden Besitzstand gemacht worden ist und noch wird, dafür bieten ein bekanntes Beispiel die "Femmes ovariennes" in Paris. Gewiss würden diese unglücklichen Wesen auch ohne ihre Ovarie nach wie vor hysterisch sein, aber sie würden doch von einem mehr oder weniger künstlich herangezogenen und schädlicher Weise in den Mittelpunkt des krankhaften Bewusstseins gesetzten Symptom frei geblieben sein. Es ist also die Frage, ob in der Einschränkung der üblichen Untersuchnng der Ovarialgegend Hysterischer auf das nothwendigste Maass eine ärztliche Pflicht zu erblicken ist; jedenfalls wird der Versuch einer solchen Einschränkung nicht als unausführbar bezeichnet werden können. Handelt es sich doch um die Verhtitung ganz ähnlicher Nachtheile, wie solche z. B. auf dem Gebiet der Hypnose als Folge unnöthiger Manipulationen und unbedachter Suggestionen bereits allgemein anerkannt sind. Ueberdies steht die Vornahme in häufigen Fällen mindestens überflüssiger Handlungen und die Ueberschreitung des Nothwendigen bei der Untersuchung im Widerspruch mit dem zum Vortheil der Kranken geübten (Bruns $\left.{ }^{24}\right)$ ) und heute als werthvolles psychotherapeutisches Hülfsmittel mehr und mehr anerkannten systematischen Ignoriren.

Eine weitere Frage wäre die, ob die Untersuchung der Ovarialgegend wie auch anderer hysterogener Zonen zur Diagnose thatsächlich nothwendig sei. Dass die Ovarieerscheinungen bis zu einem gewissen und recht beträchtlichen Grade diagnostisch überhaupt nicht zu verwerthen sind, glaube ich nachgewiesen zu haben, und da, wo Erscheinungen in hysterischer Steigerung auftreten, wird die Diagnose der Hysterie stets schor us anderweitigen Indicien hinreichend sicher zu stellen sein. Seltene Ausnahmen mag man zugeben! - Schliesslich möchte ich noch hemerken, dass auch der Umstand die Integrität meines Untersuchu, smaterials beweist, dass in keinem Fall irgendwie der Hysterie zuzurechnende krankhafte Steigerung von Reactionserscheinungen sich bemerkbar gemacht hat. 


\section{Literatur.}

1) Schützemberger, Gazette médicale de Paris. 1846. Nr. 22, 23, 25, 39, $40,43$.

2) Briquet, Traité clinique; cit. bei Charcot.

3) Schultze, Arch. f. Gynäk. IX. S. 262.

4) Hasse, Ebenda. VIII. S. 20.

5) Schroeder, Berlin. klin. Woch. 1883. Nr. 40.

6) Gusserow, Zeitschrift f. Geb. u. Gynäk. X. S. 114.

7) Landau und Remak, Ztschr. f. klin. Med. 1883. IV. S. 437.

8) Jolly in Ziemssen's Handbuch u. s. w. XII. Bd., 2. Hälfte. S. 479.

9) Gilles de Ia Tourette, Die Hysterie. Deutsch v. Grube. 1894. S. 184ff.

10) Hirt, Pathol. u. Ther. d. Nervenkrankh. 1890. S. 425.

11) Lomer, Beurtheilung des Schmerzes in der Gynäkologie. Wiesbaden 1899.

12) Breuer und Freud, Studien über Hysterie. Wien.

13) Gowers, Handb. d. Nervenkr. III. Bd. S. 359.

14) Kyri, Verhandl. d. Gesellschaft f. Geb. und Gynäk. Breslau 1893.

15) Ziehen, Hysterie. Eulenburg's Realencyklop. Bd. XI. S. 302-383.

16) Windscheid, Neuropathol. u. Gynäk. 1897. S. 33 und Neurol. Centralbl. 1898. Nr. 23. S. 1109.

17) Böttiger, Neurol. Centralbl. 1897. S. 515.

18) Oppenheim, Lehrbuch der Nervenkrankh. 1898. 2. Aufl.

19) Strüm pell, D. Ztschr, f. Nervblk. 1899. 15. Bd. S. 254.

20) Düms, Hysterie in d. Armee. Festschr. etc. Dresden 1899 und Handbuch der Militärkrankheiten. III. Band. 1900. S. $179 \mathrm{ff}$.

21) Möbius, Neurol. Beiträge. 1894. I.

22) Neurol. Centralbl. 1897. S. 513 u. 1898. S. 475.

23) Gad, Reflexe in Eulenburg's Realencyklop. 1899. 15. Bd.

24) Bruns, Hysterie im Kindesalter. Halle 1897.

25) Goldscheider, Empfindung. Eulenburg's Realencyklop. 1898. 6. Bd. S. 625.

26) Charcot, Klin. Vorträge, übersetzt v. Fetzer.

Derselbe, Neue Vorlesungen, übers. v. Freud.

Derselbe, Poliklin. Vortr., übers. v. Freud. 